\title{
Prototyping a coherent framework for full, fast and parametric detector simulation for the FCC project
}

\author{
Julia Hrdinka ${ }^{* a}$, Anna Zaborowska ${ }^{b}$, Andreas Salzburger $^{c}$, Benedikt Hegner $^{c}$ \\ a Vienna University of Technology \\ Vienna, Austria \\ $b$ Warsaw University of Technology \\ Warsaw, Poland \\ c CERN \\ Geneva, Switzerland \\ E-mail: julia.hrdinka@cern.ch, anna.zaborowska@cern.ch, \\ andreas.salzburger@cern.ch, benedikt.hegnerdcern.ch
}

\begin{abstract}
The outstanding success of the physics program of the Large Hadron Collider (LHC) including the discovery of the Higgs boson shifted the focus of part of the high energy physics community onto the planning phase for future circular collider (FCC) projects. A proton-proton collider is in consideration, as well as an electron-positron ring and an electron-proton option as potential LHC successor projects. Common to all projects is the need for a coherent software framework in order to carry out simulation studies to establish the potential physics reach or to test different technology approaches. Detector simulation is a particularly necessary tool needed for design studies of different detector concepts and to allow establishing the relevant performance parameters. In addition, it allows to generate data as input for the development of reconstruction algorithms needed to cope with the expected future environments. We present a coherent framework that combines full, fast and parametric detector simulation embedded in the Gaudi framework and based on the FCC event data model. Detector description is based on DD4Hep and the different simulation approaches are centrally steered through the Geant 4 simulation. A prototype example of a simple tracking detector is demonstrated for the different simulation approaches and a potential work flow to use full simulation based on Geant 4 and fast simulation techniques alongside is presented.
\end{abstract}

The European Physical Society Conference on High Energy Physics

22-29 July 2015

Vienna, Austria

${ }^{*}$ Speaker. 


\section{Future Circular Collider (FCC)}

The big success of the physics program of the Large Hadron Collider (LHC) at CERN (including the discovery of the Higgs Boson) led to the planning of future collider projects. The FCC (Future Circular Collider) [1] is a five year international design study to explore post LHC possibilities until the end of 2018. A conceptual design report will be delivered as an outcome of this study.

A tunnel of a circumference of $80-100 \mathrm{~km}$ is being considered to potentially host three different high energy machines. A first step involves an electron-positron-collider (FCC-ee) with a centerof-mass energy of 90-400 GeV. Such a machine could be used for precision measurements of the recently discovered Higgs boson and the Standard Model (SM) electro-weak sector. Finally, a proton-proton collider is envisioned with a center-of-mass energy of $100 \mathrm{TeV}$ (FCC-hh). A third option is an electron-proton-collider (FCC-eh), which could operate at the same time as FCCee/FCC-hh and allows for complementary measurements and determinations of particle density functions needed for the p-p operation. One possible localization of this project could be at CERN, Switzerland. The collider could benefit from the already existing accelerator chain by using the LHC as an injector. These machines would offer the possibility to search for new physics at unprecedented energies and to study known phenomena in high precision.

\section{FCC simulation framework}

For these three rather diverse communities a common software suite is in development. The software community started by reviewing existing software solutions of the current LHC experiments and additional available resources. Special emphasis was put on the coherent combination of full and fast simulation types. Full simulation uses the full and very detailed detector geometry and simulates the physics processes precisely. However, experiences from the LHC Run-1 showed that the available computing resources already led to limitations of the Monte Carlo (MC) statistics that can be produced with a full simulation setup. Fast simulation approaches grew in importance, in particular for large statistics samples. In the following, first steps towards a simulation framework that combines full and fast simulation techniques will be presented.

Gaudi [2], which is an experiment independent software architecture and framework for high energy physics data applications was chosen as the event processing framework. The main concept of the Gaudi architecture is a scheduled sequence of algorithms that communicate via a central data store using a well defined event data model (EDM). A coherent simulation framework only unfolds its full benefits, if it is supported by such a common EDM that is shared between the different simulation approaches. The FCC event data model [3] is based on simple C++ classes and structs and could already be used for analysis. The persistification and input/output technology of the FCC EDM is based on the ROOT [4] toolkit. For detector description, the DD4Hep (Detector Description for High Energy Physics) [5] package, originating from linear collider studies, is used. Its internal geometry model is based on the TGeo package of ROOT. For the simulation Geant4 [6] was chosen as the simulation kernel. It handles both, the standard full simulation as well as the fast simulation, taking advantage of its internal region based fast simulation capabilities. 


\subsection{A coherent simulation framework}

To facilitate the analysis and the comparison of the data, a coherent simulation framework that allows the combination of the different simulation types is essential. This simulation framework has a single input for the detector geometries (DD4hep) needed by the different simulation types and an identical analysis event data model. We aim to create a flexible framework, following the approach of the ATLAS ISF (Integrated Simulation Framework) [7]. The basic idea of the ISF is the encapsulation of the actual particle simulation from the simulation steering, allowing to attach different simulation techniques (fast/full/parametric) to certain regions of the detector or even depending on event topologies. Thus, accuracy and speed can be balanced in different combinations, using the detailed full simulation where needed and a fast simulation for regions of less interest for physics studies. The main difference to the ISF is that the region based mixing of simulation techniques is done using Geant 4 as the simulation kernel rather than a separate framework. This kernel is receiving the particles and sends them to the various simulations defined in a certain detector region for a certain particle.

Figure 1 shows the motivation for the data flow of the combined Monte Carlo simulation chain including fast, full and parametric simulation. The user steers the simulation and interacts with the

Data flow

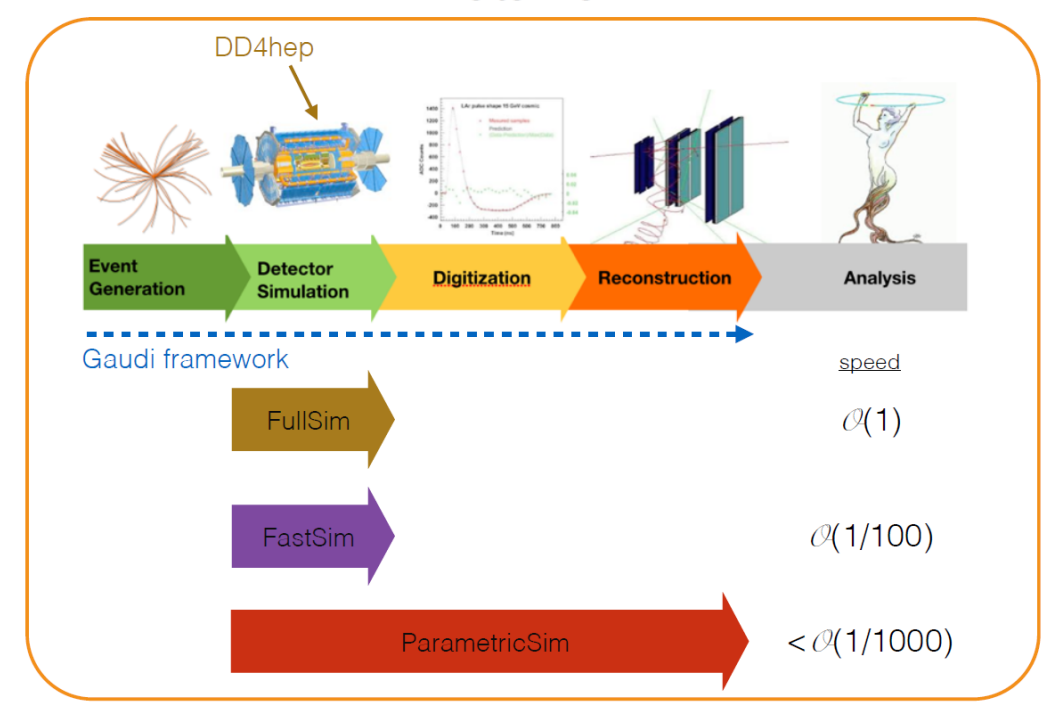

Figure 1: Motivation for the FCC simulation framework. DD4hep is the single source of detector description. After an event is generated, it is sent to the particular simulations configured within the Gaudi framework. The simulation is followed by digitization and reconstruction, before the data can be used for analysis. In the lower part, the different simulation types established are shown and their speed is compared, the speed estimates are taken from experiences with the ATLAS Integrated Simulation Framework.

framework from Gaudi over job configuration files. DD4hep is used as single source of geometry input. After the event is generated by an event generator the particles are sent to the different kinds of detector simulations. Digitization simulates the detector response, that aims to transform the plain simulation input into signals as being read out by the detector readout system. The last step is the reconstruction, which describes the set of algorithms that are responsible to build meaningful 
physics objects, such as particles or jets from the plain detector input.

\subsection{Detector simulation}

The full simulation is completely done in the Geant 4 environment which usually uses a very detailed detector geometry. An automatic translation from the DD4hep geometry into the Geant4 geometry is part of the DD4hep toolkit. The Geant4 simulation is the most accurate detector simulation to date and makes a step by step transportation of the particle through the material. The high accuracy has the drawback of high CPU consumption and a low speed, therefore fast simulation approaches were implemented into the framework.

Fast simulation techniques try to emulate the full simulation output by applying different approaches. They usually implement simplifications or parameterisations of the full simulation effects. The simplification can be done for the geometry, the physics processes or can be implemented by skipping certain steps or parameterising the whole process.

In the parametric simulation approach the different detector regions are described by simple envelopes. Different fast simulation models are attached to these detector regions, using the parameters obtained from full simulation as input. The particle is transported from the entry to the exit point of the envelope, while its measured parameters (usually energy, momentum or track parameters) are smeared with correlated smearing functions. For each detector model, new resolutions need to be obtained from the full simulation, however this can be done with a reasonable smaller number of events. Currently two approaches of the parametric simulation are implemented: a flexible parametric simulation integrated into the Geant 4 framework and a parametric simulation based on the Delphes [8] toolkit.

Fast track simulation often uses the reconstruction geometry (as e.g. realized by the ATLAS and CMS collaborations). The reconstruction geometry is usually a simplified detector geometry, consisting of the sensitive material and a simplified material description for the rest of the detector. In this way the simulation is much faster but less precise. However, it still reflects the detector effects accurately. The concepts and functionalities of the ATLAS reconstruction geometry [9] were adapted to fit into the FCC software framework, keeping the main concept of connective navigation, while allowing a direct translation from the full DD4Hep geometry. This was achieved by making a construction from TGeo volumes possible. The DD4hep readout segmentation is used in this geometry for coherency reasons. A navigation is embedded into this geometry to enable material effects in reconstruction and enable fast simulation. An automated transcript was introduced which translates the full into the reconstruction geometry.

\section{First tests of full, fast and parametric simulation}

In order to test the parametric simulation in the Geant4 environment, the resolution file of the ATLAS Technical Design Report (TDR) was used to reproduce the residual plots and the corresponding gaussian standard deviations, obtained from fits of the residual functions. Figure 2 shows a comparison of the original ATLAS plots with the recreated distributions within the FCC framework. 

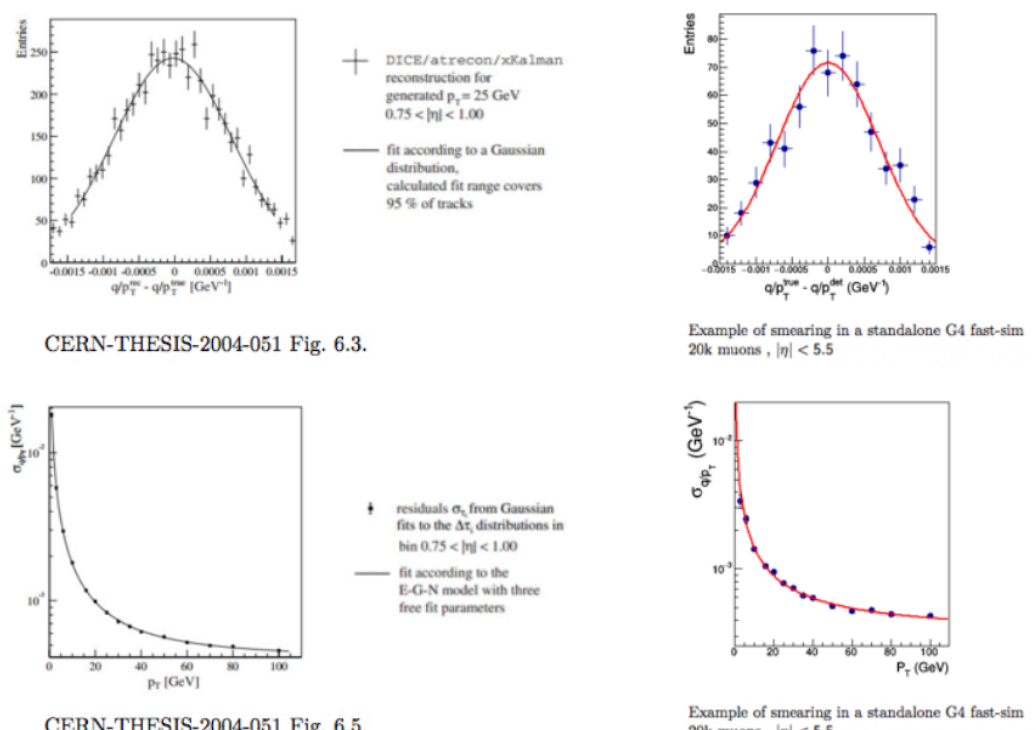

Figure 2: Smearing of $q / p_{T}$ of 20000 muons with a transverse momentum $p_{T}=25 \mathrm{GeV}$. Tested against ATLAS TDR resolution file. Left: original plots of ATLAS. Right: reproduced plots by the parametric simulation for FCC.

To test the translation from the full into the simplified reconstruction geometry a first simple test tracking detector was introduced. During the translation into the reconstruction geometry, the geometry is automatically simplified. Each detector module is approximated as a surface, while the material parameters are averaged over its components. The translation also ensures to enable the navigation, which creates relations between the different geometry objects, so that a continuous movement through the detector is possible. Each geometrical object can be accessed at every point of the detector. Inside one volume this is realized by packing the volumes fully with layers, which can hold the detector modules or are empty and only serve for navigation. These layers point to their previous/next layer. To navigate between the volumes, these are bordered by boundary surfaces, which point to their previous/next volumes. To test the navigation and to validate the geometry a hit comparison between the full and the fast simulation was done. The detector modules were intersected randomly in a straight line, beginning from the detector center and after displaying the points, a tomography of the sensitive detector can be made. Furthermore the material (thickness in units of the radiation length) seen in $\eta$ in the full simulation is compared with material seen in fast simulation (see Fig 3).

\section{Summary and outlook}

A simulation framework to combine full, fast and parametric detector simulation was successfully established. It is possible to run three different kinds of simulation types from one geometry input and configuration setup. The geometry can be provided in the Geant 4 and the reconstruction geometry within the framework.

The next steps will be the completion of the FCC simulation suite and the improvement of the 

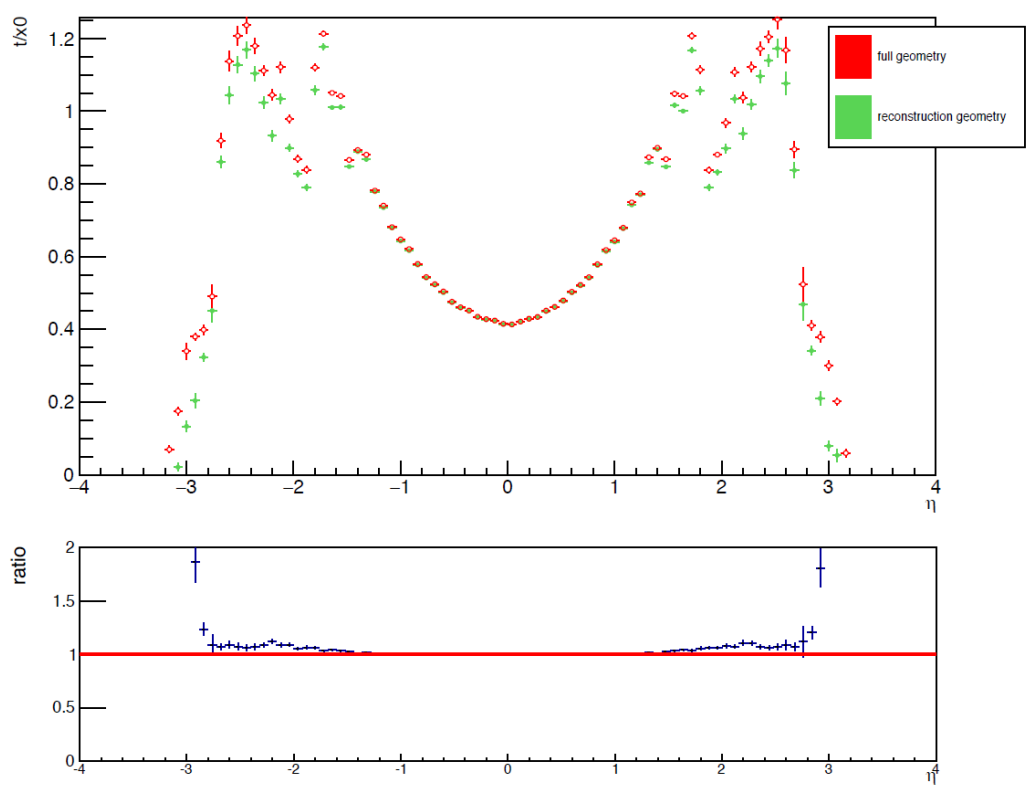

Figure 3: Material budget (thickness in units of the radiation length) over $\eta$ seen by fast simulation, which uses the reconstruction geometry and seen in full simulation, which uses the full geometry.

simulations. The automatic transcript will be improved to provide a more flexible translation. A transport through the magnetic field will be implemented for the fast simulation and reconstruction algorithms will be integrated from the LHC experiments. The integration of single particle reconstruction into the Gaudi framework to obtain resolutions for the parametric simulation with a FCC detector model will be done. A further prospective is the expansion of the simulations for a use in calorimeters.

\section{References}

[1] FCC webpage, http:// cern.ch/fcc

[2] Gaudi webpage, http://cern.ch/gaudi

[3] FCC Event Data Model Twiki page,

https://twiki.cern.ch/twiki/bin/viewauth/FCC/FCCSoftwareEDM

[4] Rootwebpage, https://root.cern.ch

[5] DD4hep webpage, http://aidasoft.web.cern.ch/DD 4 hep

[6] Geant4 wegpage, https://geant 4 .web.cern.ch/geant 4

[7] E. Ritsch, ATLAS Detector Simulation in the Integrated Simulation Framework applied to the W Boson Mass Measurement, Universitaet Innsbruck, 2014, http://physik.uibk.ac.at/hephy/theses/diss_er.pdf,

[8] Delphes simulation webpage, https://cp3.irmp.ucl.ac.be/projects/delphes,

[9] A. Salzburger, S. Todorova, M. Wolter, The ATLAS Tracking Geometry Description, The ATLAS Collaboration, CERN, 2007, [ATL-SOFT-PUB-2007-004] 